\title{
Uncontrolled intracellular osmotic pressure leads to cancer
}

\author{
Feng Zhang ${ }^{1}$, Runmin Jiang ${ }^{2}$, Chao Zhang ${ }^{3}$ \\ ${ }^{1}$ State Key Laboratory of Cancer Biology, Department of Pathology of Xijing \\ Hospital and School of Basic Medicine, Fourth Military Medical University, \\ Xi'an 710038, China \\ ${ }^{2}$ Department of Thoracic Surgery, Tangdu Hospital, Fourth Military Medical \\ University, Xi'an 710038, China. \\ ${ }^{3}$ Department of Urology, No. 904 Hospital of the PLA Joint Logistic Support \\ Force, Wuxi 214000, China.
}

${ }^{1}$ Corresponding author. email: zhf1975@fmmu.edu.cn

Key words: Cell division, Intracellular osmotic pressure; Tolerance limit of cell membrane; Carcinogenesis; Aneuploidy; $\mathrm{Na}^{+} / \mathrm{K}^{+}$pump; Cytoskeleton, Oncogene; Tumor suppressor 
Abstract

At present more than 9 million people die of cancer every year. Simple and broad-spectrum drugs are still an urgent need for cancer patients. Recently, we proposed a new hypothesis that intracellular osmotic pressure (IOP) is the driving force of cell division, and abnormal tumor proliferation is the result of uncontrolled IOP in cells. On the one hand, aneuploidy and abnormal function of $\mathrm{Na}^{+} / \mathrm{K}^{+}$pump lead to a faster rise of IOP in tumor cell than normal cells, on the other hand, abnormality of cytoskeleton assembly leads to the decrease of tolerance limit of cell membrane (TLCM) of tumor cells for resisting IOP. This hypothesis predicts: 1)Tumor cells were more intolerant to hypotonic stress than normal cells. 2) Maligancies may be sellectively killed by a suddenn increase of IOP and combined with decrease of the TLCM of tumors. $\mathrm{Na}^{+} / \mathrm{K}^{+}$pump inhibitors can promotely increase the IOP of tumor cells and cytoskeleton inhibitors can dramatically lower the TLCM of tumor cells. Therefore, $\mathrm{Na}^{+} / \mathrm{K}^{+}$pump and cytoskeleton inhibitors may have a synergetic effect to kill tumor cells. 3) Molecules regulating cell osmolality may be new targets for cancer treatment. 
The latest statistics show that 18 million new cancer cases and 9.6 million deaths occur each year in the world (1). At present, targeted drugs and immunotherapy have made great breakthroughs(2). However, due to the heterogeneity of gene mutations of tumor cells $(3,4)$, molecular diagnosis and targeted therapy have become more and more complicated. Moreover, there are no specific targeted drugs for quite a number of malignant tumors (4-6). So, simple and broad-spectrum cancer treatment is still an urgent need.

Tumor cells are characterized by abnormal proliferation. Deep understanding of the mechanism of cell proliferation is the basis of exploring the mechanism of carcinogenesis. Traditional theory views that growth factor is the promoter of cell proliferation ${ }^{(7)}$. However, there are still some problems that cannot be explained. For example, the growth of bacteria and plants is not as dependent on growth factors as mammalian cells ${ }^{(8,9)}$, so there may be another explanation for the mechanism of cell proliferation.

Recently, we proposed a new hypothesis that uncontrolled intracellular osmotic pressure (IOP) leads to cancer based on the semi permeability of cell membrane ${ }^{(10)}$,This hypothesis holds that IOP is the driving force of cell proliferation, and abnormal tumor proliferation is the result of uncontrolled IOP of cells. We believe that verification of this hypothesis may not only deepen our understanding of the mechanism of cell proliferation and carcinogenesis, but could also explore new methods for cancer treatment.

\section{IOP is the driving force of cell division}


Cell structure is complex, which often confuses us. Recently, we try to understand the mechanism of cell division physically. The cell membrane is a lipid bimolecular layer, which could form a spherical structure with a diameter of about $10-30 \mathrm{~nm}$ in water. The cell membrane is semi permeable. Water molecules and lipids pass through the cell membrane more easily than macromolecules and ions (11, 12). Because the molar concentration of substances inside cells is generally greater than outside cells, the IOP is generally greater than extracellular osmotic pressure (EOP) of cells. Indeed, the IOP in bacteria, fungi and plants often reaches $4-5$ atmospheric pressure ${ }^{(9,}$ ${ }^{13,14)}$. What's more, contrary to the general view that the IOP and EOP of mammalian cells are equal. Some phenomena suggest that the IOP and EOP of mammalian cells is not naturally equal. For example, the cells will swell for a short time of ischemia and hypoxia, and If longer, the cell could even necrosis. Therefore, the maintaining balance of the IOP and EOP of mammalian cells rely on energy consumption. In fact, mammalian cells mainly rely on cytoskeleton actin and $\mathrm{Na}^{+} / \mathrm{K}^{+}$pump to resist IOP, and the assembly of actin and the work of $\mathrm{Na}^{+} / \mathrm{K}^{+}$pump require energy consumption ${ }^{(15-17)}$. In vitro experiments show that $20-70 \%$ of the ATP in cells is used to maintain the balance of IOP and EOP of mammalian cells ${ }^{(15,16)}$. Therefore, if mammalian cells do not actively regulate the balance of IOP and EOP, they will burst quickly like soap bubbles. Only by controlling the the balance of IOP and EOP, the cells may not be swollen and necrotic. On the other hand, since IOP has 
such a great power, it is possible for cells to use the power of IOP to complete the process of cell division, and as a result IOP is no longer the destructive force but the power of cell division(10). This hypothesis is supported by the experiment of AFM: the pressure on the cell membrane increases continuously during the process of cell division, rising from the G0 stage to the highest point at the $\mathrm{M}$ stage. With cell division, the pressure drops rapidly (17). Therefore, we believed that increase of IOP is the driving force of mammalian cell division. The fundamental reason for periodically division of cells is to periodically lower the IOP.

\section{Increase of IOP promotes cell swell and cell division}

If IOP is considered to be the driving force of cell division, it must meet at least two conditions: 1) promotion of cell division and 2) initiation of DNA replication. Early studies have shown that bacteria, fungi, and plant cells swell before they start to divide, and the power of swelling comes from IOP $(13,14$, 18-20). Recently, it has been found that in the process of mammalian cell division, the mammalian cells also swell at first and then divide $(17,21)$. It is interesting that the volume of cells after division is close to that before division. What causes cells to keep the same volume? Because the value of tolerance limit of cell membrane (TLCM) is constant, when IOP reaches the value of TLCM, cells must divide, if not, they will burst and die. Therefore, cell division may only be an active way to avoid bursting before division. In this way, when IOP rises to the value of TLCM, it is the time point of cell division. In the same 
environment, the value of TLCM of same type cells is near, so the size of these cells is similar. Similarly, in different environments, different types of cells have different TLCM, so there are differences in cell size. Therefore, increase of IOP promotes cell swell and cell division. In fact, some experiments indirectly support this hypothesis. For example, proper low osmolarity stress can promote cell proliferation(22), and the low concentration of $\mathrm{Na}^{+} / \mathrm{K}^{+}$pump inhibitor can intiate DNA replication of neuron (23), while the high osmolarity environment inhibits cell proliferation (24).

\section{Increase of IOP expand nuclear space for initiation of DNA replication}

The existence of binucleate, multinucleated cells and meiosis suggest that DNA replication and cell division are two independent events, both theoretically and experimentally $(25,26)$. However, it is clear that only when a synergistic relationship between DNA replication and cell division is established can cells survive. For most cells, DNA must complete at least one round of replication before cell division. However, does DNA replication initiate cell division or does cell division initiate DNA replication? If DNA replication initiates cell division, DNA replication should not be less than one cycle before cell division. So there should be cells with multiple cycles of replication. However, up to now, almost all eukaryotic cells, including fungal, plant and animal cells, have only one cycle of replication before each cell division. Although in a nutrient-rich environment, bacterial cells can initiate multiple rounds of replication before division, only one round of replication is completed 
before cell division (13). Therefore, DNA replication initiated by cell division may be more reasonable. How does cell division start DNA replication? There may be at least two mechanisms, one is chemical signaling mechanism and the other is physical mechanics mechanism. As IOP promotes cell volume expansion, the membrane will release chemical stress signals under mechanical pressure, and the stress signals will increase with the rise of IOP. These chemical stress signals will initiate DNA replication through signal pathway. In fact,substantial experimental evidence support this suggestion ${ }^{(27)}$.

Under pathological conditions, the cell nucleus will shrink due to the contraction of nuclear membrane and nuclear matrix. For example, apoptotic and necrotic cells show nuclear pyknosis. Therefore, in physiological state, IOP may play an important role in providing the physical space for DNA to function. Nuclear space of eukaryotic cells is limited. Without extra space, DNA may not be able to replicate (28-31). Therefore, IOP promotes the cell volume to expand during the process of cell division, meanwhile, through the cytoskeleton to pull the cell nuclear membrane, promotes the cell nuclear volume to increase, provides space for DNA replication. Therefore, DNA replication needs not only a certain concentration of chemical stress signals, but also the physical space. In addition, it was found that bacterial DNA replication initiation sites are directly linked to cell membrane, while eukaryotic DNA replication initiation sites are linked to nuclear skeleton or nuclear matrix $(32,33)$. Because DNA replication needs to open DNA double strands, there 
may be a possibility: the expanding volume of cell directly or indirectly pulling DNA through the cytoskeleton and nuclear matrix, which may help to open DNA double strands and trigger DNA replication. Although there is no evidence that IOP can directly initiate DNA replication, a large number of experiences shows that physical traction can promote cell division, such as skin traction to expand the area of skin graft $(34,35)$. Therefore, the increase of IOP not only provides physical space for DNA replication, but also may directly initiate DNA replication by mechanical pull. In conclusion, IOP is the driving force of cell division because it can promote cell division and initiate DNA replication.

\section{IOP and cytoskeleton regulate cell differentiation by changing cell}

\section{morphology}

Limited space can limit DNA replication, as well as RNA transcription. Although it is clear that the production of different types of cells during development is regulated by signal molecules and transcription factors (36), the maintenance of different cell types is controlled by epigenetic modifications $(37,38)$. However, it is not clear whether physical factors could affect gene transcription. Animal cell types are diverse, each of which has different three-dimensional geometry. The difference of cell morphology indicates that the cell nucleus is pulled by the cytoskeleton in various directions of three-dimensional, which leads to different shapes of nucleus ${ }^{(39)}$. Many results showed that the binding site of DNA and nuclear matrix was active in gene transcription ${ }^{(40)}$, therefore, it can not be ruled out that IOP and cytoskeleton 
work together to regulate the shape of nucleus, resulting in different physical space sizes in different directions, thus affecting gene transcription level in different directions of DNA, and ultimately affecting cell differentiation. If so, the constant changes in cell morphology during development may also play an important role in the production and maintenance of cell types. Therefore, IOP and cytoskeleton can not only change cell morphology, but also indirectly affect gene transcription and cell differentiation $(41,42)$. Recently, it was found that the genes of transcriptional activation in nucleus will move from transcriptional silencing region to transcriptional activation region(43). A typical example is Hox gene clusters. Using $4 \mathrm{C}$-seq, the Duboule laboratory observed that the activated hox genes shift from an inactive chromatin domain to an active domain during the development the embryonic axis(44). Although the mechanism of position change of Hox gene is not clear, we speculate that it may be the result of physical traction with nuclear morphological change.

\section{Delaying or inhibiting cell division costs more energy}

In a nutrient-rich environment, the cell cycle of bacteria is usually 40 minutes but that of plant cells takes more than 20 hours. The thickness of bacterial cell wall is about $10 \mathrm{~nm}$, while that of plant cell is at least $100 \mathrm{~nm}(9$, $13,14)$. This suggests that delaying cell division requires greater endurance to fight the rise of IOP. In line with this, inhibiting mammalian cell proliferation also requires more energy. Mammalian cells are generally divided into three types. The first is unstable cells, such as skin and intestinal epithelial cells, which are 
always in a state of division. The second is stable cells, such as liver cells, which are generally not obviously divided. The third is permanent cells, such as cardiomyocytes and neurons, that is, in any case, they no longer divide. Mammalian cells generally adopt two ways to control IOP. The first is cytoskeleton, which fight against the swelling force caused by IOP in cell. The second is $\mathrm{Na}^{+} / \mathrm{K}^{+}$pump, which pump out three sodium ions and in two potassium ions at a time, lowering the ionic osmotic pressure inside the cell, and slowing down the increase of the overall IOP. Substantial evidence have shown that the level of unstable cell membrane potential is low $(10-30 \mathrm{mV})$, while that of the stable cell membrane potential is medium $(40-50 \mathrm{mV})$, and that of the permanent cell membrane potential is the highest $(60-70 \mathrm{mV})$. The membrane potential reflects the energy consumption against IOP by $\mathrm{Na}^{+} / \mathrm{K}^{+}$ pump ${ }^{(45,46)}$. Therefore, it takes more energy to inhibit or slow down cell division.

\section{Uncontrolled IOP of cells leads to cancer}

Cancer is a disease of uncontrolled cell proliferation. When IOP reach to TLCM, the cell begins to divide, and if not, it will burst and die $(10,13)$. Therefore, the speed of cell division is mainly determined by two factors: the acceleration of IOP and the value of TLCM. If there exists any factors which could increase the acceleration of IOP and/or decrease the value of TLCM, then the cells would divide prematurely. Due to the time of DNA synthesis and repair cannot be shortened at will, premature division of cells before 
completion of DNA replication or repair would lead to increased gene mutation rate, genomic instability, uneven chromosome distribution, karyotype aneuploidy, vicious cycle, and finally cancer. In fact, many studies demonstrated that the number of DNA replication initiation sites in tumor cells increase significantly compared with normal cells, suggesting that tumor cells are indeed in a state of premature division(47-51). Therefore, many genetic changes in tumor cells can be attributed to premature division caused by uncontrolled IOP.

There are at least two lines of evidence showing that the acceleration of IOP in tumor cells is greater than that in normal cells: 1) the membrane potential of tumor cells is lower than that of normal cells ${ }^{(45,52)}$. Moreover, many studies have shown that the concentration of $\mathrm{Na}^{+}$in tumor cells is $2-5$ times higher than that in normal cells ${ }^{(52-54)}$. These indicated that the function of $\mathrm{Na}^{+} / \mathrm{K}^{+}$pump in tumor cells decrease. 2) Most tumor cells are aneuploid karyotype, and the number of chromosomes is generally more than that of normal cells $(55,56)$. In equal time, aneuploidy produces more RNA and protein and increase the acceleration of IOP in tumor cells compared with normal cells, thus initiating tumor cell division and DNA replication in advance $(10,57)$

On the other hand, many lines of evidence show that the value of TLCM of tumor cells to resist IOP is decreased. 1) Histological and morphological studies showed that the cytoskeleton of tumor cells was obviously abnormal 
(58-68). 2) Transgenic experiment showed that oncogene products such as Src, Ras and Rho could directly disrupt the assembly of cytoskeleton ${ }^{(69-71)}$. 3) Some studies have shown that tumor suppressor protein RB is a nuclear matrix junction protein and linked with DNA replication initiation site (72-74). Moreover, P53 and Rb were demonstrated to inhibit tumorigenesis by remodeling cytoskeleton $(75,76) .4)$ The common contradictory phenomenon observed in clinical tumor pathological sections is that, with the higher grade of malignancy, not only the percentage of proliferative tumor cells and multinucleated tumor giant cells but also the number of necrotic tumor cells increase(77-80). Our explanation is that rise of IOP drives tumor cells to divide, however, the division of mother tumor cell into two daughter tumor cells require the contraction force of M-phase actomyosin contraction ring should be greater than $\operatorname{IOP}(10,17,81,82)$. The more malignant the tumor is, the more serious the function of actin is damaged $(58-62,64,65,83)$. Therefore, with the increase of tumor malignancy, the decrease of TLCM results in more tumor cells in the state of division, but on the other hand, decrease of contraction ring function don't overcome IOP and fail to divide mother tumor cell into two daughter tumor cells, resulting increasing number of multinucleated tumor giant cells or massive necrosis.

In conclusion, we believe that carcinogenesis is the result of uncontrolled IOP. Moreover, due to the alteration of $\mathrm{Na}^{+} / \mathrm{K}^{+}$pump, cytoskeleton and nuclear matrix of malignant tumor cells, the morphological changes will affect 
gene transcription through physical and mechanical mechanism, so the differentiation of malignant tumor is abnormal.

\section{Targeted IOP regulatory molecules to treat malignant tumors}

The new hypothesis have some predictions as following. 1) Tumor cells are more intolerant of hypotonic stress than normal cells. Although few people directly compare the tolerance between tumor cells and normal cells to hypotonic stress, the application of distilled water to lavage the chest and abdominal cavity after clinical operation can significantly reduce the spread and metastasis of malignancies and improve the survival rate of patients $(84$, 85). The survival time of mice inoculated with distilled water was significantly longer than that of normal saline (86). 2) Penicillin mainly inhibits the synthesis of cell wall, resulting in bacteria unable to withstand the IOP and death $(13,87)$. We think similar idea may also be used in the treatment of malignant tumors. Compared with normal cells, IOP of tumor cells increases faster and TLCM value is lower. Therefore, we think that if we target regulating molecules of IOP to further accelerate IOP and reduce TLCM value, it is possible to selectively kill tumor cells. As inhibition of $\mathrm{Na}^{+} / \mathrm{K}^{+}$pump can dramatically increase IOP and inhibition of cytoskeleton can reduce TLCM, the combination of $\mathrm{Na}^{+} / \mathrm{K}^{+}$pump and cytoskeleton inhibitors may have a stronger synergistic effect to kill tumor cells. At present, there are more than ten kinds of inhibitors of $\mathrm{Na}^{+} / \mathrm{K}^{+}$pump and cytoskeleton respectively(88-96). In recent years, many anti-tumor drug screening experiments have proved that digoxin has obvious anti-tumor effect 
(97-99), and many cytoskeleton inhibitors have been used in clinic for many years (100). Therefore, from these inhibitors, we may be able to screen effective drug combinations for cancer treatment. 3) molecules of IOP regulation may be a new target for cancer treatment.

Acknowledgments: This work was supported by the National Natural Science Foundation of China (81572631 and 31000559) and Shaanxi Society Development Sci-Tech Research Project (2016SF-064) to F.Z.

Conflict of Interest: The authors declare that they have no conflict of interest.

Author contribution: F.Z. had the idea for the article and performed the literature search and data analysis. F. Z., R.M.J. and C.Z. critically revised the work.

\section{References}

[1] F. Bray, J. Ferlay, I. Soerjomataram, R.L. Siegel, L.A. Torre and A. Jemal, Global cancer statistics 2018: GLOBOCAN estimates of incidence and mortality worldwide for 36 cancers in 185 countries, CA Cancer J Clin 68 (2018), pp. 394-424.

[2] J. Nangalia and P.J. Campbell, Genome Sequencing during a Patient's Journey through Cancer, N Engl J Med 381 (2019), pp. 2145-2156.

[3] I. Dagogo-Jack and A.T. Shaw, Tumour heterogeneity and resistance to cancer therapies, Nat Rev Clin Oncol 15 (2018), pp. 81-94.

[4] B. Vogelstein, N. Papadopoulos, V.E. Velculescu, S. Zhou, L.A. Diaz, Jr. and K.W. Kinzler, Cancer genome landscapes, Science 339 (2013), pp. 1546-1558.

[5] M. Gerstung, C. Jolly, I. Leshchiner, et al., The evolutionary history of 2,658 cancers, Nature 578 (2020), pp. 122-128.

[6] Y. Li, N.D. Roberts, J.A. Wala, et al., Patterns of somatic structural variation in human cancer genomes, Nature 578 (2020), pp. 112-121.

[7] D. Hanahan and R.A. Weinberg, Hallmarks of cancer: the next generation, Ce// 144 (2011), 
pp. 646-674.

[8] P. Nurse, A long twentieth century of the cell cycle and beyond, Cell 100 (2000), pp. $71-78$.

[9] D. Kierzkowski and A.L. Routier-Kierzkowska, Cellular basis of growth in plants: geometry matters, Curr Opin Plant Bio/ 47 (2019), pp. 56-63.

[10] F. Zhang, A new hypothesis about cell division and carcinogenesis(In Chinese), Negative 10 (2019), p. 3.

[11] S.J. Singer and G.L. Nicolson, The fluid mosaic model of the structure of cell membranes, Science 175 (1972), pp. 720-731.

[12] G.L. Nicolson, The Fluid-Mosaic Model of Membrane Structure: still relevant to understanding the structure, function and dynamics of biological membranes after more than 40 years, Biochim Biophys Acta 1838 (2014), pp. 1451-1466.

[13] A.L. Koch, How bacteria grow and divide in spite of internal hydrostatic pressure, Can J Microbio/ 31 (1985), pp. 1071-1084.

[14] M. Osawa and H.P. Erickson, Turgor Pressure and Possible Constriction Mechanisms in Bacterial Division, Front Microbio/9 (2018), p. 111.

[15] M. Muller, W. Siems, F. Buttgereit, R. Dumdey and S.M. Rapoport, Quantification of ATP-producing and consuming processes of Ehrlich ascites tumour cells, Eur J Biochem 161 (1986), pp. 701-705

[16] B.W. Bernstein and J.R. Bamburg, Actin-ATP hydrolysis is a major energy drain for neurons, J Neurosci 23 (2003), pp. 1-6.

[17] M.P. Stewart, J. Helenius, Y. Toyoda, S.P. Ramanathan, D.J. Muller and A.A. Hyman, Hydrostatic pressure and the actomyosin cortex drive mitotic cell rounding, Nature 469 (2011), pp. 226-230.

[18] N. Minc, A. Boudaoud and F. Chang, Mechanical Forces of Fission Yeast Growth, Curr Biol 24 (2014), p. 1436.

[19] R. Sablowski and M. Carnier Dornelas, Interplay between cell growth and cell cycle in plants, J Exp Bot 65 (2014), pp. 2703-2714.

[20] E.R. Rojas and K.C. Huang, Regulation of microbial growth by turgor pressure, Curr Opin Microbio/ 42 (2018), pp. 62-70.

[21] E. Zlotek-Zlotkiewicz, S. Monnier, G. Cappello, M. Le Berre and M. Piel, Optical volume and mass measurements show that mammalian cells swell during mitosis, J Cel/ Bio/ 211 (2015), pp. 765-774.

[22] M. Gonczi, N. Szentandrassy, L. Fulop, et al., Hypotonic stress influence the membrane potential and alter the proliferation of keratinocytes in vitro, Exp Dermato/ 16 (2007), pp. 302-310.

[23] C.D. Cone, Jr. and C.M. Cone, Induction of mitosis in mature neurons in central nervous system by sustained depolarization, Science 192 (1976), pp. 155-158.

[24] A. Dascalu, A. Matithyou, Y. Oron and R. Korenstein, A hyperosmotic stimulus elevates intracellular calcium and inhibits proliferation of a human keratinocyte cell line, $J$ Invest Dermato/ 115 (2000), pp. 714-718.

[25] R. Bernander and K. Nordstrom, Chromosome replication does not trigger cell division in E. coli, Cel/ 60 (1990), pp. 365-374.

[26] A.L. Koch, Does the initiation of chromosome replication regulate cell division?, $A d v$ 
Microb Physio/ 16 (1977), pp. 49-98.

[27] B. Ekundayo and F. Bleichert, Origins of DNA replication, PLoS Genet 15 (2019), p. e1008320.

[28] C.J. Cattin, M. Duggelin, D. Martinez-Martin, C. Gerber, D.J. Muller and M.P. Stewart, Mechanical control of mitotic progression in single animal cells, Proc Natl Acad Sci U S A 112 (2015), pp. 11258-11263.

[29] J. Folkman and A. Moscona, Role of cell shape in growth control, Nature 273 (1978), pp. 345-349.

[30] S. Huang, C.S. Chen and D.E. Ingber, Control of cyclin D1, p27(Kip1), and cell cycle progression in human capillary endothelial cells by cell shape and cytoskeletal tension, Mol Biol Cel/ 9 (1998), pp. 3179-3193.

[31] S.J. Streichan, C.R. Hoerner, T. Schneidt, D. Holzer and L. Hufnagel, Spatial constraints control cell proliferation in tissues, Proc Natl Acad Sci U S A 111 (2014), pp. 5586-5591.

[32] D.A. Jackson and P.R. Cook, Replication occurs at a nucleoskeleton, EMBO J5 (1986), pp. 1403-1410.

[33] G.B. Ogden, M.J. Pratt and M. Schaechter, The replicative origin of the E. coli chromosome binds to cell membranes only when hemimethylated, Cell 54 (1988), pp. 127-135.

[34] L. LeGoff and T. Lecuit, Mechanical Forces and Growth in Animal Tissues, Cold Spring Harb Perspect Bio/ 8 (2015), p. a019232.

[35] S.A. Gudipaty, J. Lindblom, P.D. Loftus, et al., Mechanical stretch triggers rapid epithelial cell division through Piezo1, Nature 543 (2017), pp. 118-121.

[36] J. Deschamps and D. Duboule, Embryonic timing, axial stem cells, chromatin dynamics, and the Hox clock, Genes Dev 31 (2017), pp. 1406-1416.

[37] A. Piunti and A. Shilatifard, Epigenetic balance of gene expression by Polycomb and COMPASS families, Science 352 (2016), p. aad9780.

[38] B. Schuettengruber, H.M. Bourbon, L. Di Croce and G. Cavalli, Genome Regulation by Polycomb and Trithorax: 70 Years and Counting, Ce// 171 (2017), pp. 34-57.

[39] K.C. Clause, L.J. Liu and K. Tobita, Directed stem cell differentiation: the role of physical forces, Cell Commun Adhes 17 (2010), pp. 48-54.

[40] A. Feuerborn and P.R. Cook, Why the activity of a gene depends on its neighbors, Trends Genet 31 (2015), pp. 483-490.

[41] F.M. Watt, P.W. Jordan and C.H. O'Neill, Cell shape controls terminal differentiation of human epidermal keratinocytes, Proc Natl Acad Sci U S A 85 (1988), pp. 5576-5580.

[42] M.C. Jones, J. Zha and M.J. Humphries, Connections between the cell cycle, cell adhesion and the cytoskeleton, Philos Trans R Soc Lond B Biol Sci 374 (2019), p. 20180227.

[43] W. de Laat and D. Duboule, Topology of mammalian developmental enhancers and their regulatory landscapes, Nature 502 (2013), pp. 499-506.

[44] D. Noordermeer, M. Leleu, E. Splinter, J. Rougemont, W. De Laat and D. Duboule, The dynamic architecture of Hox gene clusters, Science 334 (2011), pp. 222-225.

[45] C.D. Cone, Jr., Variation of the transmembrane potential level as a basic mechanism of mitosis control, Oncology 24 (1970), pp. 438-470.

[46] M. Yang and W.J. Brackenbury, Membrane potential and cancer progression, Front Physio/ 4 (2013), p. 185. 
[47] D. Di Paola, E. Rampakakis, M.K. Chan, D.N. Arvanitis and M. Zannis-Hadjopoulos, Increased origin activity in transformed versus normal cells: identification of novel protein players involved in DNA replication and cellular transformation, Nucleic Acids Res 38 (2010), pp. 2314-2331.

[48] A. Oppenheim and R.G. Martin, Initiation points for DNA replication in nontransformed and simian virus 40-transformed BALB/c 3T3 cells, J Viro/ 25 (1978), pp. 450-452.

[49] D. Di Paola, G.B. Price and M. Zannis-Hadjopoulos, Differentially active origins of DNA replication in tumor versus normal cells, Cancer Res 66 (2006), pp. 5094-5103.

[50] R.G. Martin and A. Oppenheim, Initiation points for DNA replication in nontransformed and simian virus 40-transformed Chinese hamster lung cells, Cel/ 11 (1977), pp. 859-869.

[51] J.S. Butel and H.R. Soule, Role of the simian virus 40 gene A product in regulation of DNA synthesis in transformed cells, J Viro/ 26 (1978), pp. 584-594.

[52] I. Nagy, G. Lustyik, G. Lukacs, V. Nagy and G. Balazs, Correlation of malignancy with the intracellular $\mathrm{Na}+\mathrm{K}+$ ratio in human thyroid tumors, Cancer Res 43 (1983), pp. 5395-5402.

[53] I.Z. Nagy, G. Lustyik, V.Z. Nagy, B. Zarandi and C. Bertoni-Freddari, Intracellular Na+:K+ ratios in human cancer cells as revealed by energy dispersive $\mathrm{x}$-ray microanalysis, $\mathrm{J}$ Cell Bio/ 90 (1981), pp. 769-777.

[54] I. Nagy, L. Toth, Z. Szallasi and I. Lampe, Energy-dispersive, bulk specimen X-ray microanalytical measurement of the intracellular $\mathrm{Na}+/ \mathrm{K}+$ ratio in human laryngeal tumors, J Cancer Res Clin Oncol 113 (1987), pp. 197-202.

[55] H. Rajagopalan and C. Lengauer, Aneuploidy and cancer, Nature 432 (2004), pp. 338-341.

[56] P. Duesberg, Does aneuploidy or mutation start cancer?, Science 307 (2005), p. 41.

[57] H.J. Tsai, A.R. Nelliat, M.I. Choudhury, et al., Hypo-osmotic-like stress underlies general cellular defects of aneuploidy, Nature 570 (2019), pp. 117-121.

[58] R. Pollack, M. Osborn and K. Weber, Patterns of organization of actin and myosin in normal and transformed cultured cells, Proc Natl Acad Sci U S A 72 (1975), pp. 994-998.

[59] E. Rungger-Brandle and G. Gabbiani, The role of cytoskeletal and cytocontractile elements in pathologic processes, Am J Patho/ 110 (1983), pp. 361-392.

[60] E. Friedman, M. Verderame, S. Winawer and R. Pollack, Actin cytoskeletal organization loss in the benign-to-malignant tumor transition in cultured human colonic epithelial cells, Cancer Res 44 (1984), pp. 3040-3050.

[61] G. Pawlak and D.M. Helfman, Cytoskeletal changes in cell transformation and tumorigenesis, Curr Opin Genet Dev 11 (2001), pp. 41-47.

[62] V. Swaminathan, K. Mythreye, E.T. O'Brien, A. Berchuck, G.C. Blobe and R. Superfine, Mechanical stiffness grades metastatic potential in patient tumor cells and in cancer cell lines, Cancer Res 71 (2011), pp. 5075-5080.

[63] B.B. Asch, D. Medina and B.R. Brinkley, Microtubules and actin-containing filaments of normal, preneoplastic, and neoplastic mouse mammary epithelial cells, Cancer Res 39 (1979), pp. 893-907.

[64] E. Friedman, M. Verderame, M. Lipkin and R. Pollack, Altered actin cytoskeletal patterns in two premalignant stages in human colon carcinoma development, Cancer Res 45 (1985), pp. 3236-3242. 
[65] S.M. Haghparast, T. Kihara, Y. Shimizu, S. Yuba and J. Miyake, Actin-based biomechanical features of suspended normal and cancer cells, J Biosci Bioeng 116 (2013), pp. 380-385.

[66] J. Tang, J.W. Niu, D.H. Xu, Z.X. Li, Q.F. Li and J.A. Chen, Alteration of nuclear matrix-intermediate filament system and differential expression of nuclear matrix proteins during human hepatocarcinoma cell differentiation, World J Gastroentero/ 13 (2007), pp. 2791-2797.

[67] S. Prasad, V.A. Soldatenkov, G. Srinivasarao and A. Dritschilo, Intermediate filament proteins during carcinogenesis and apoptosis (Review), Int J Oncol 14 (1999), pp. 563-570.

[68] P. Bannasch, H. Zerban and D. Mayer, The cytoskeleton in tumor cells, Pathol Res Pract 175 (1982), pp. 196-211.

[69] J. Rao and N. Li, Microfilament actin remodeling as a potential target for cancer drug development, Curr Cancer Drug Targets 4 (2004), pp. 345-354.

[70] J.C. Barros and C.J. Marshall, Activation of either ERK1/2 or ERK5 MAP kinase pathways can lead to disruption of the actin cytoskeleton, J Cel/ Sci 118 (2005), pp. 1663-1671.

[71] A. Hall, The cytoskeleton and cancer, Cancer Metastasis Rev 28 (2009), pp. 5-14.

[72] M.A. Mancini, B. Shan, J.A. Nickerson, S. Penman and W.H. Lee, The retinoblastoma gene product is a cell cycle-dependent, nuclear matrix-associated protein, Proc Natl Acad Sci U S A 91 (1994), pp. 418-422.

[73] T. Ozaki, M. Saijo, K. Murakami, H. Enomoto, Y. Taya and S. Sakiyama, Complex formation between lamin $A$ and the retinoblastoma gene product: identification of the domain on lamin A required for its interaction, Oncogene 9 (1994), pp. 2649-2653.

[74] T.A. Kim, J. Lim, S. Ota, et al., NRP/B, a novel nuclear matrix protein, associates with p110(RB) and is involved in neuronal differentiation, J Cel/ Bio/141 (1998), pp. 553-566.

[75] T. Ebata, H. Hirata and K. Kawauchi, Functions of the Tumor Suppressors p53 and Rb in Actin Cytoskeleton Remodeling, Biomed Res Int 2016 (2016), p. 9231057.

[76] K. Araki, T. Ebata, A.K. Guo, K. Tobiume, S.J. Wolf and K. Kawauchi, p53 regulates cytoskeleton remodeling to suppress tumor progression, Cell Mol Life Sci 72 (2015), pp. 4077-4094.

[77] B. Delahunt, J.N. Eble, L. Egevad and H. Samaratunga, Grading of renal cell carcinoma, Histopathology 74 (2019), pp. 4-17.

[78] S. Rathore, T. Niazi, M.A. Iftikhar and A. Chaddad, Glioma Grading via Analysis of Digital Pathology Images Using Machine Learning, Cancers (Basel) 12 (2020).

[79] E.A. Rakha, M. Aleskandarani, M.S. Toss, et al., Breast cancer histologic grading using digital microscopy: concordance and outcome association, J Clin Pathol 71 (2018), pp. 680-686.

[80] M. Trojani, G. Contesso, J.M. Coindre, et al., Soft-tissue sarcomas of adults; study of pathological prognostic variables and definition of a histopathological grading system, Int J Cancer 33 (1984), pp. 37-42.

[81] C. Huang, Y. Li and H. Wang, The observation of high hypotonicity manipulating cell division, Heliyon 5 (2019), p. e02095.

[82] I. Mendes Pinto, B. Rubinstein and R. Li, Force to divide: structural and mechanical requirements for actomyosin ring contraction, Biophys J105 (2013), pp. 547-554.

[83] C. Alibert, B. Goud and J.B. Manneville, Are cancer cells really softer than normal cells?, 
Biol Cel/ 109 (2017), pp. 167-189.

[84] L. Rajdev, Treatment options for surgically resectable gastric cancer, Curr Treat Options Onco/ 11 (2010), pp. 14-23.

[85] A. Shiozaki, D. Ichikawa, T. Kosuga, Y. Marunaka and E. Otsuji, Regulation of osmolality for cancer treatment, J Physiol Sci 67 (2017), pp. 353-360.

[86] A. Shiozaki, D. Ichikawa, K. Takemoto, et al., Efficacy of a hypotonic treatment for peritoneal dissemination from gastric cancer cells: an in vivo evaluation, Biomed Res Int 2014 (2014), p. 707089.

[87] A.L. Koch, The exocytoskeleton, J Mol Microbiol Biotechno/ 11 (2006), pp. 115-125.

[88] T. Mijatovic, F. Dufrasne and R. Kiss, Na+/K+-ATPase and cancer, Pharm Pat Anal 1 (2012), pp. 91-106.

[89] C.T. Durlacher, K. Chow, X.W. Chen, et al., Targeting $\mathrm{Na}(+) / \mathrm{K}(+)$-translocating adenosine triphosphatase in cancer treatment, Clin Exp Pharmacol Physio/ 42 (2015), pp. 427-443.

[90] I. Prassas and E.P. Diamandis, Novel therapeutic applications of cardiac glycosides, Nat Rev Drug Discov 7 (2008), pp. 926-935.

[91] A.L. Risinger and L. Du, Targeting and extending the eukaryotic druggable genome with natural products: cytoskeletal targets of natural products, Nat Prod Rep (2019).

[92] M. Trendowski, Exploiting the cytoskeletal filaments of neoplastic cells to potentiate a novel therapeutic approach, Biochim Biophys Acta 1846 (2014), pp. 599-616.

[93] K. Haider, S. Rahaman, M.S. Yar and A. Kamal, Tubulin inhibitors as novel anticancer agents: an overview on patents (2013-2018), Expert Opin Ther Pat 29 (2019), pp. 623-641.

[94] A. Nurnberg, A. Kollmannsperger and R. Grosse, Pharmacological inhibition of actin assembly to target tumor cell motility, Rev Physiol Biochem Pharmacol 166 (2014), pp. $23-42$.

[95] P. Sima and V. Vetvicka, Bioactive substances with anti-neoplastic efficacy from marine invertebrates: Porifera and Coelenterata, World J Clin Onco/ 2 (2011), pp. 355-361.

[96] S.Y. Saito, Toxins affecting actin filaments and microtubules, Prog Mol Subcel/ Bio/ 46 (2009), pp. 187-219.

[97] S. Gkountela, F. Castro-Giner, B.M. Szczerba, et al., Circulating Tumor Cell Clustering Shapes DNA Methylation to Enable Metastasis Seeding, Cell 176 (2019), pp. 98-112 e114.

[98] E.A. Platz, S. Yegnasubramanian, J.O. Liu, et al., A novel two-stage, transdisciplinary study identifies digoxin as a possible drug for prostate cancer treatment, Cancer Discov 1 (2011), pp. 68-77.

[99] C.D. Simpson, I.A. Mawji, K. Anyiwe, et al., Inhibition of the sodium potassium adenosine triphosphatase pump sensitizes cancer cells to anoikis and prevents distant tumor formation, Cancer Res 69 (2009), pp. 2739-2747.

[100] J. Tischer and F. Gergely, Anti-mitotic therapies in cancer, J Cell Biol 218 (2019), pp. $10-11$. 